\title{
Undiagnosed Peripheral Nerve Disease in Patients with Failed Lumbar Disc Surgery
}

\author{
Tomohiro Yamauchi ${ }^{1}$, Kyongsong $\mathrm{Kim}^{1,2}$, Toyohiko Isu ${ }^{1}$, Naotaka Iwamoto ${ }^{3}$, \\ Kazuyoshi Yamazaki ${ }^{1}$, Juntaro, Matsumoto ${ }^{1}$, Masanori Isobe ${ }^{1}$ \\ ${ }^{1}$ Department of Neurosurgery, Kushiro Rosai Hospital, Hokkaido, Japan \\ ${ }^{2}$ Department of Neurosurgery, Chiba Hokuso Hospital, Nippon Medical School, Chiba, Japan \\ ${ }^{3}$ Department of Neurosurgery, Teikyo University School of Medicine, Tokyo, Japan
}

Study Design: Retrospective study (level of evidence=3).

Purpose: We examine the relationship between residual symptoms after discectomy for lumbar disc herniation and peripheral nerve (PN) neuropathy.

Overview of Literature: Patients may report persistent or recurrent symptoms after lumbar disc herniation surgery; others fail to respond to a variety of treatments. Some PN neuropathies elicit symptoms similar to those of lumbar spine disease.

Methods: We retrospectively analyzed data for 13 patients treated for persistent ( $n=2)$ or recurrent $(n=11)$ low back pain (LBP) and/or leg pain after primary lumbar discectomy.

Results: Lumbar re-operation was required for four patients (three with recurrent lumbar disc herniation and one with lumbar canal stenosis). Superior cluneal nerve (SCN) entrapment neuropathy (EN) was noted in 12 patients; SCN block improved the symptoms for eight of these patients. In total, nine patients underwent PN surgery (SCN-EN, $n=4$; peroneal nerve $E N, n=3$; tarsal tunnel syndrome, $\mathrm{n}=1$ ). Their symptoms improved significantly.

Conclusions: Concomitant PN disease should be considered for patients with failed back surgery syndrome manifesting as persistent or recurrent LBP.

Keywords: Low back pain; Peripheral nerve; Lumbosacral region; Intervertebral disc disease; Nerve compression syndrome

\section{Introduction}

Lumbar degenerative disease is often treated by lumbar surgery, with lumbar discectomy, which is a commonly used surgical procedure to address lumbar disc herniation (LDH). However, some patients who have been operated report persistent or recurrent symptoms. This is known as failed back surgery syndrome (FBSS), and it has been attributed to recurrent disc herniation, epidural fibrosis or hematoma, inadequate decompression of coexisting segmental stenosis, segmental instability, dural tear, and/ or psychosocial factors [1-6].

Some peripheral neuropathies elicit symptoms similar to those of lumbar spine disease [7]. For example, superior cluneal nerve $(\mathrm{SCN})$ entrapment neuropathy $(\mathrm{SCN}$ EN), a causative factor in low back pain (LBP) that is induced and exacerbated by lumbar movement, can be misdiagnosed as a lumbar spine disorder [8-12]. Similarly,

Received Oct 23, 2017; Revised Dec 5, 2017; Accepted Dec 17, 2017

Corresponding author: Kyongsong Kim

Department of Neurosurgery, Chiba Hokuso Hospital, Nippon Medical School, 1715, Kamagari, Inzai city, Chiba, Japan

Tel: +81-476-99-1111, Fax: +81-476-99-1906, E-mail: kyongson@nms.ac.jp 
common peroneal nerve (CPN) entrapment neuropathy (CPN-EN) elicits leg symptoms similar to those of lumbar disease; therefore, a differential diagnosis is important [7,13-16]. Peripheral nerve (PN) diseases following lumbar discectomy can potentially be overlooked. Therefore, in this study, we examined the relationship between residual or persistent symptoms following primary discectomy for $\mathrm{LDH}$ and concomitant $\mathrm{PN}$ disease.

\section{Materials and Methods}

Our study was approved by the institutional ethics committee of Kushiro Rosai Hospital (no. 28-3); prior written informed consent was obtained from all patients included in this study.

\section{Patients}

This retrospective study included 13 patients with persistent $(n=2)$ or recurrent ( $n=11)$ LBP and/or leg pain, whose LDH had been treated by primary discectomy between April 2013 and May 2015. They included eight men and five women, with a mean age of 58.2 years (range, 3378 years). All had undergone primary lumbar discectomy for $\mathrm{LDH}$, five at our institution and eight elsewhere. None presented with trauma around the knee, malignancy, active infection, Parkinson's disease, dementia, or psychological issues. The LDH level was L4/5 in 11 patients and L5/S1 in the other two. The mean interval between symptom exacerbation and presentation at our department was 12 months (range, 1-60 months); the mean postoperative follow-up period was 26.4 months (range, 9-39 months) (Table 1). Acetaminophen, non-steroidal anti-inflammatory drugs, and/or tramadol failed to control their pain; none were given codeine or morphine.

\section{Criteria for the diagnosis of superior cluneal nerve- entrapment neuropathy}

We carefully assessed the patients to confirm that SCN entrapment played a role in their LBP using established diagnostic criteria $[11,12,17]$. LBP was considered to be the result of SCN-EN if there was unilateral pain involving the iliac crest and buttocks, there was a trigger point over the posterior iliac crest located $7 \mathrm{~cm}$ from the midline (corresponding to the nerve compression zone), and the patient experienced numbness and radiating pain in the SCN area when the trigger point was compressed. Most patients experienced symptom relief within 2 hours of the administration of a local nerve block ( $2 \mathrm{~mL}$ of $1 \%$ lidocaine injected at the trigger point in the buttock); however, some reported the recurrence of pain after the analgesic effect of the injection wore off. SCN was blocked with a minimal amount of local analgesic to avoid misdiagnosis of the origin of the patient's pain.

\section{Criteria for the diagnosis of common peroneal nerve- entrapment neuropathy}

The diagnosis of CPN-EN was based on symptoms including numbness and/or pain from the lower leg to the dorsum of the foot, which appeared when the patient walked or stood and resulted in intermittent claudication $[13,15,16]$. All patients were positive for Tinel's sign at the $\mathrm{CPN}$ around the neck of the fibula.

\section{Criteria for the diagnosis of tarsal tunnel syndrome}

Tarsal tunnel syndrome (TTS) results from entrapment of the posterior tibial nerve in the tarsal tunnel on the medial malleolus. Its diagnosis is based on symptoms such as numbness and/or pain in the sole of the foot, without heel involvement, which appear when the patient walks or stands for a prolonged time $[7,18,19]$. All the patients with TTS were positive for Tinel's sign at the tarsal tunnel.

\section{Evaluation of treatment outcomes}

The treatment outcomes were assessed on a numeric rating scale (NRS) and with the Roland-Morris Disability Questionnaire (RDQ). The scores were recorded before treatment and at the latest follow-up. The data were assessed with paired $t$-tests using StatMate III software (ATMS Co. Ltd., Tokyo, Japan). Differences of $p<0.05$ were considered statistically significant.

\section{Results}

The findings for the 13 patients with recurrent or persistent pain are summarized in Table 1. In three of the patients (cases 1-3), the pain recurred at the level of the LDH. In another patient (case 4), the pain was due to lumbar spinal canal stenosis at the operated level. No abnormal findings were observed in lumbar magnetic reso- 


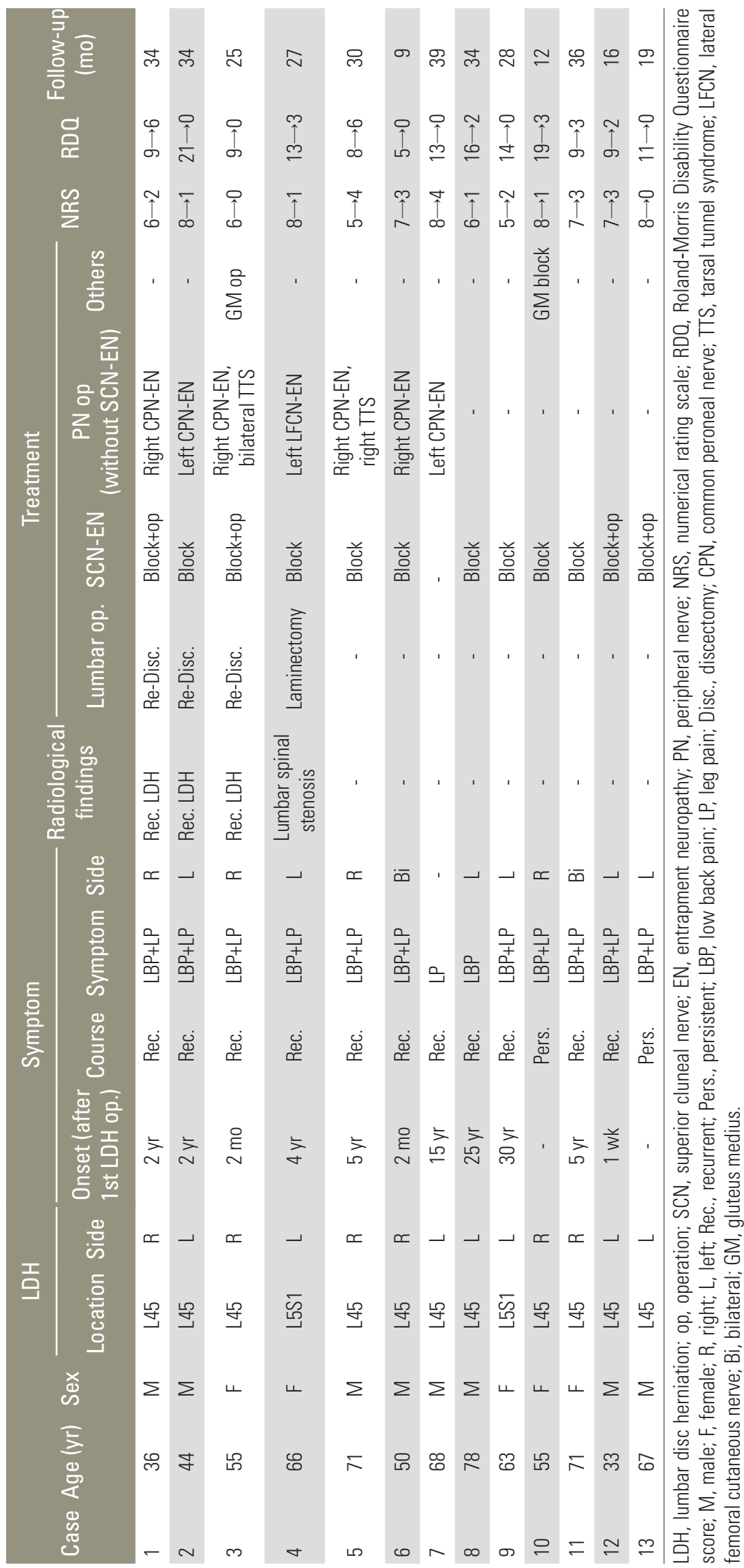


nance imaging and computed tomography studies for the other nine patients.

Additionally, 11 patients reported both LBP and leg pain; one patient suffered only LBP, and one experienced only leg pain. The symptoms recurred a median of 2 years (range, 0-30 years) after the first LDH surgery. In four patients (cases 1-4), we observed radiological abnormalities indicative of lower limb radiculopathy along the dermatome; all had previously undergone same level lumbar surgery. These patients required lumbar re-operation.

SCN-EN was diagnosed in 12 patients; in 10 of these, the SCN-EN was unilateral and in nine of these the symptom was on the same side as the lumbar lesion. Of these, two patients (cases 10,13) reported persistent ipsilateral pain after the first LDH surgery, and the symptoms of another patient (case 12) reappeared within the first week after the first LDH operation. SCN block was effective in eight patients, who did not undergo SCN-EN surgery. SCN-EN surgery was required for four patients.

PN rather than SCN-EN surgery was required for seven patients (CPN-EN, $n=6$; TTS, $n=2$; lateral femoral cutaneous nerve entrapment, $n=1$ ). Of these, four patients (cases 1-4) required lumbar re-operation: two patients (cases 1,2) underwent PN surgery to address residual leg symptoms attributable to CPN-EN, one patient (case 3), who reported residual symptoms, was operated to address CPN-EN, bilateral TTS, and gluteus medius muscle (GMM) pain, and another patient (case 4) with residual symptoms was operated for left lateral femoral cutaneous nerve EN. The three other patients (cases 5-7) required CPN-EN surgery; patient of case 5 also needed TTS surgery to address leg symptoms. The treatments were successful for all but one of the patients (case 5), with significantly improved NRS scores $(6.8-1.9, p<0.05)$ and RDQ scores $(12.0-1.9, p<0.05)$ (Table 1$)$.

\section{Discussion}

\section{Failed back surgery syndrome after lumbar disc her- niation surgery}

Although surgical treatment for LDH can deliver good results, $5 \%-40 \%$ of operated patients manifest FBSS [1-6]. Of these, $3 \%-18 \%$ suffer recurrence of the $\mathrm{LDH}[4,5,20]$. Patients with FBSS but no recurrence of the LDH have been observed to exhibit epidural fibrosis, epidural hematoma, inadequate decompression of coexisting segmental stenosis, segmental instability, dural tear, and/or arachnoiditis [1-6]. Long-lasting back pain has been reported by $10 \%-30 \%$ of patients after primary discectomy for radiculopathy [21-23]. Consequently, the potential causes of postoperative pain must be considered in patients with FBSS manifesting as LBP after LDH surgery.

We evaluated and treated 13 patients with persistent or recurrent symptoms after LDH surgery. Radiological studies confirmed LDH recurrence in three of the patients and lateral recess stenosis in one patient; they underwent additional lumbar decompression surgery. The other nine patients manifested no radiological abnormalities that explained their persistent or recurrent symptoms. However, treatment for PN neuropathy resulted in symptom abatement in these patients, suggesting that factors other than lumbar disease were involved. We believe that the persistent postoperative symptoms of two patients with LDH (cases 10,13) were due to coexisting PN neuropathy, which had not been addressed during the LDH surgery. Our findings indicate that concomitant PN disease should be considered in patients with persistent or recurrent symptoms after LDH surgery.

\section{Symptoms due to superior cluneal nerve-entrapment neuropathy}

Previously, we have documented the usefulness of less invasive treatments for PN neuropathy in patients with LBP and leg pain $[8-13,16,18]$. These symptoms are similar to those elicited by lumbar disease. The presence of SCN-EN in $1.4 \%-14 \%$ of patients is higher than is commonly expected [8-12,17]. Of our 13 patients with FBSS manifesting as persistent LBP, 12 required treatment for SCN-EN, with SCN block and SCN neurolysis proving beneficial. According to earlier reports, SCN block successfully addressed SCNEN in $28 \%-100 \%$ of patients, even in those with severe LBP $[12,17,24]$. In the present study, eight of the 12 patients with FBSS reported abatement or disappearance of their pain after SCN blockage, suggesting that this procedure should be considered for patients with LBP after LDH surgery.

In nine of the 10 patients with unilateral symptoms due to SCN-EN, the symptoms were on the same side as the lumbar lesion. The sinuvertebral nerves around vertebral structures such as the outer annulus fibrosus of the intervertebral discs contribute to the elicitation of pain around the spine, including discogenic or vertebral joint pain [25]. Although the pathogenesis of SCN-EN remains unknown, 
stimulation of the sinuvertebral nerve by LDH and/or unilateral nerve root irritation caused by radiculopathy may play a role, and SCN-EN may be misdiagnosed as lumbar herniation in patients with LBP.

\section{Symptoms due to other peripheral nerve diseases}

Out of the 13 patients, six underwent neurolysis for CPNEN. This is the most common PN neuropathy of the lower limb and the most frequent cause of leg numbness and pain involving the affected area without severe paresis or intermittent claudication [13-16,26]. Because PN neuropathy can manifest symptoms similar to those of L5 radiculopathy due to lumbar lesions, a differential diagnosis is important, even after LDH surgery. CPN-EN can be treated by less invasive neurolysis under local anesthesia. However, the diagnosis of CPN-EN can be difficult because it cannot always be detected electrophysiologically; careful symptom assessment may yield a correct diagnosis. Previously, we have reported that the repetitive plantar flexion test is a useful provocation test for diagnosing CPN-EN $[13,14,16]$.

TTS is an EN of the posterior tibial nerve within the tarsal tunnel. The symptoms are sensory disturbance in the sole of the foot without heel involvement, paresthesia, and a sensation of coldness or the presence of a foreign body $[7,18,19]$. The symptoms are exacerbated by prolonged standing or walking and the condition may be misdiagnosed as spinal disease. TTS has been observed in $4.8 \%$ of patients with lumbosacral radiculopathy [27], suggesting it may underlie the residual symptoms in patients with FBSS.

GMM is located in the buttock, partially beneath the gluteus maximus muscle. It can be the origin of buttock pain with lateral and/or posterior femoral pain $[28,29]$. The diagnosis of GMM pain is based on clinical symptoms. Treatment consists of GMM block and decompression surgery under local anesthesia $[28,29]$. As with lumbar disease, the pain is exacerbated by sitting, standing, and walking; a differential diagnosis in patients reporting LBP requires the identification of the underlying cause. Of our patients, two required treatment for GMM pain. Although GMM pain is not reflective of PN neuropathy, the latter should be considered for patients with residual symptoms due to FBSS.

\section{Study limitations}

This study had some limitations. The study population was small, the follow-up period was short (mean, 26.7 months), and the interval between surgery and symptom recurrence varied among the patients. The symptoms were persistent in some and recurrent in others, and they manifested immediately after, soon after, or a long time after surgery. In addition, this was a retrospective study and not all patients underwent their first operation at our institution. Consequently, our findings may not accurately reflect the incidence of true PN neuropathy in patients with FBSS after LDH surgery. We do not know what proportion of the total number of patients operated during our study period manifested FBSS, and we cannot comment on the overall effectiveness of surgical PN treatment. Nonetheless, we recommend that spinal surgeons consider PN disease for patients with FBSS.

\section{Conclusions}

We successfully treated patients with persistent or recurrent pain after LDH surgery. Our findings highlight the importance of considering PN symptoms in these patients. Less invasive treatment for PN neuropathy should be considered.

\section{Conflict of Interest}

No potential conflict of interest relevant to this article was reported.

\section{References}

1. Chan CW, Peng P. Failed back surgery syndrome. Pain Med 2011;12:577-606.

2. Fiume D, Sherkat S, Callovini GM, Parziale G, Gazzeri G. Treatment of the failed back surgery syndrome due to lumbo-sacral epidural fibrosis. Acta Neurochir Suppl 1995;64:116-8.

3. Rohde V, Mielke D, Ryang Y, Gilsbach JM. The immediately failed lumbar disc surgery: incidence, aetiologies, imaging and management. Neurosurg Rev 2015;38:191-5.

4. Shriver MF, Xie JJ, Tye EY, et al. Lumbar microdiscectomy complication rates: a systematic review and meta-analysis. Neurosurg Focus 2015;39:E6.

5. Skaf G, Bouclaous C, Alaraj A, Chamoun R. Clinical outcome of surgical treatment of failed back surgery syndrome. Surg Neurol 2005;64:483-8. 
6. Wilson CA, Roffey DM, Chow D, Alkherayf F, Wai EK. A systematic review of preoperative predictors for postoperative clinical outcomes following lumbar discectomy. Spine J 2016;16:1413-22.

7. Kim K, Isu T, Morimoto D, et al. Common diseases mimicking lumbar disc herniation and their treatment. Mini-Invasive Surg 2017;1:43-51.

8. Chiba Y, Isu T, Kim K, et al. Association between intermittent low-back pain and superior cluneal nerve entrapment neuropathy. J Neurosurg Spine 2015 Oct 13 [Epub]. https://doi.org/10.3171/2015.1.SPINE14173.

9. Iwamoto N, Isu T, Kim K, et al. Low back pain caused by superior cluneal nerve entrapment neuropathy in patients with Parkinson disease. World Neurosurg 2016;87:250-4.

10. Kim K, Isu T, Chiba Y, et al. Treatment of low back pain in patients with vertebral compression fractures and superior cluneal nerve entrapment neuropathies. Surg Neurol Int 2015;6(Suppl 24):S619-21.

11. Morimoto D, Isu T, Kim K, et al. Surgical treatment of superior cluneal nerve entrapment neuropathy. J Neurosurg Spine 2013;19:71-5.

12. Kuniya H, Aota Y, Kawai T, Kaneko K, Konno T, Saito T. Prospective study of superior cluneal nerve disorder as a potential cause of low back pain and leg symptoms. J Orthop Surg Res 2014;9:139.

13. Iwamoto N, Kim K, Isu T, Chiba Y, Morimoto D, Isobe M. Repetitive plantar flexion test as an adjunct tool for the diagnosis of common peroneal nerve entrapment neuropathy. World Neurosurg 2016;86:484-9.

14. Kim K, Isu T, Kokubo R, Morimoto D, Kobayashi S, Morita A. Repetitive plantar flexion (provocation) test for the diagnosis of intermittent claudication due to peroneal nerve entrapment neuropathy: case report. NMC Case Rep J 2015;2:140-2.

15. Maalla R, Youssef M, Ben Lassoued N, Sebai MA, Essadam H. Peroneal nerve entrapment at the fibular head: outcomes of neurolysis. Orthop Traumatol Surg Res 2013;99:719-22.

16. Morimoto D, Isu T, Kim K, et al. Microsurgical decompression for peroneal nerve entrapment neuropathy. Neurol Med Chir (Tokyo) 2015;55:669-73.

17. Maigne JY, Doursounian L. Entrapment neuropathy of the medial superior cluneal nerve: nineteen cases surgically treated, with a minimum of 2 years' followup. Spine (Phila Pa 1976) 1997;22:1156-9.
18. Kim K, Isu T, Morimoto D, et al. Neurovascular bundle decompression without excessive dissection for tarsal tunnel syndrome. Neurol Med Chir (Tokyo) 2014;54:901-6.

19. Kokubo R, Kim K, Isu T, et al. The impact of tarsal tunnel syndrome on cold sensation in the pedal extremities. World Neurosurg 2016;92:249-54.

20. Ambrossi GL, McGirt MJ, Sciubba DM, et al. Recurrent lumbar disc herniation after single-level lumbar discectomy: incidence and health care cost analysis. Neurosurgery 2009;65:574-8.

21. Parker SL, Mendenhall SK, Godil SS et al. Incidence of low back pain after lumbar discectomy for herniated disc and its effect on patient-reported outcomes. Clin Orthop Relat Res 2015;473:1988-99.

22. Parker SL, Xu R, McGirt MJ, Witham TF, Long DM, Bydon A. Long-term back pain after a single-level discectomy for radiculopathy: incidence and health care cost analysis. J Neurosurg Spine 2010;12:178-82.

23. McGirt MJ, Ambrossi GL, Datoo G, et al. Recurrent disc herniation and long-term back pain after primary lumbar discectomy: review of outcomes reported for limited versus aggressive disc removal. Neurosurgery 2009;64:338-44.

24. Ermis MN, Yildirim D, Durakbasa MO, Tamam C, Ermis OE. Medial superior cluneal nerve entrapment neuropathy in military personnel; diagnosis and etiologic factors. J Back Musculoskelet Rehabil 2011;24:137-44.

25. Morimoto D, Isu T, Kim K, et al. Long-term outcome of surgical treatment for superior cluneal nerve entrapment neuropathy. Spine (Phila Pa 1976) 2017;42:783-8.

26. Mitra A, Stern JD, Perrotta VJ, Moyer RA. Peroneal nerve entrapment in athletes. Ann Plast Surg 1995;35:366-8.

27. Zheng C, Zhu Y, Jiang J, et al. The prevalence of tarsal tunnel syndrome in patients with lumbosacral radiculopathy. Eur Spine J 2016;25:895-905.

28. Kim K, Isu T, Chiba Y, Iwamoto N, Morimoto D, Isobe M. Decompression of the gluteus medius muscle as a new treatment for buttock pain: technical note. Eur Spine J 2016;25:1282-8.

29. Kokubo R, Kim K, Isu T, et al. Superior cluneal nerve entrapment neuropathy and gluteus medius muscle pain: their effect on very old patients with low back pain. World Neurosurg 2017;98:132-9. 